\title{
Effect of Loading Mode on the Evolution of the Dislocation Structure in Magnesium
}

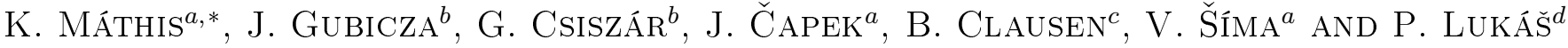 \\ ${ }^{a}$ Department of Physics of Materials, Faculty of Mathematics and Physics, Charles University, \\ Ke Karlovu 5, 12116 Prague, Czech Republic \\ ${ }^{b}$ Department of Materials Physics, Faculty of Science, Eötvös Loránd University, \\ Pázmány P. sétány 1/A, H-1117 Budapest, Hungary \\ ${ }^{c}$ Los Alamos National Laboratory, Lujan Neutron Scattering Center, Los Alamos, NM 87545, USA \\ ${ }^{d}$ Nuclear Physics Institute of the Academy of Sciences of the Czech Republic, 25068 Rež, Czech Republic
}

The evolution of the dislocation structure in randomly textured cast magnesium as a function of loading mode is studied using whole neutron diffraction pattern line profile analysis and elasto-plastic self-consistent modeling. Both the experimental results and the theoretical data indicate the onset of basal slip at low stresses and the key role of prismatic slip in the macroscopic yield. Dependence of the second-order pyramidal slip on the loading mode is revealed.

DOI: 10.12693/APhysPolA.128.700

PACS: 81.05.Bx, 83.85.Hf, 83.60.La, 81.40.Lm

\section{Introduction}

The study of the unique deformation behavior of magnesium alloys, related to their hexagonal closed packed (hcp) structure and with nearly ideal ratio of the crystallographic parameters $c / a$, represents a topical subject in the recent literature. Microstructural and experimental conditions for the activation of both basal and non-basal slip and various twinning mechanisms have been thoroughly studied [1-4]. Various theoretical models from crystal-plasticity models to atomistic calculations have been worked out for the description of the basic mechanisms of twinning, dislocation slip and twindislocation interactions [5-7]. The majority of the published experimental studies are based on ex situ techniques (e.g. microscopy methods, electron backscattered diffraction (EBSD), X-ray diffraction line profile analysis). In situ techniques, such as acoustic emission [8], digital image correlation [9] or X-ray and neutron diffraction (ND) measurements [10] during deformation tests have been successfully used for the determination of twinned volume in different magnesium alloys. Nevertheless, there are only few, recent papers dealing with the in situ investigation of the dislocation structure by ND method [11, 12], providing the largest statistical ensemble, particularly for coarse grained materials. The present work deals with the study of the dislocation structure evolution as a function of the applied loading mode (tension or compression) in randomly textured cast magnesium. Quantitative information about the particular slip systems is given using the ND profile anal-

* corresponding author; e-mail: mathis@met.mff.cuni.cz ysis. The experimental results are compared with the theoretical predictions of the elasto-plastic self-consistent (EPSC) model.

\section{Experimental procedures and modeling}

\subsection{In situ neutron diffraction experiments}

Randomly textured cast polycrystalline magnesium, having a grain size of $(100 \pm 5) \mu \mathrm{m}$ was used for the testing. Cylindrical specimens with a length of $20 \mathrm{~mm}$ and a diameter of $9 \mathrm{~mm}$ were deformed in tension and compression at a strain rate of $10^{-3} \mathrm{~s}^{-1}$ in strain control mode at room temperature using a horizontal $250 \mathrm{kN}$ capacity load frame. The angle between the longitudinal axis of the sample and the incident beam in the SMARTS engineering instrument ${ }^{\dagger}$ was $45^{\circ}$ [13]. Two detector banks were positioned at $\pm 90^{\circ}$ to the incident beam in order to record diffraction patterns in both parallel (referred as axial detector) and perpendicular (radial detector) to the loading direction. The ND patterns were recorded at predefined strain levels $(0,2,4$ and $6 \%)$ for $60 \mathrm{~min}$.

\subsection{EPSC modeling}

The EPSC deformation model [14] with an implemented twinning scheme [5] and extended to finite strains [15] was used for theoretical description of dislocation slip and mechanical twinning on the mesoscopic (grain) level. For the evolution of the instantaneous critical resolved shear stress (CRSS) of the particular slip or

\footnotetext{
$\dagger$ The SMARTS (spectrometer for materials research at temperature and stress) is a third-generation neutron diffractometer optimized for the study of engineering materials under stress and extreme temperature.
} 
twinning system $\tau^{s}$ with the total accumulated plastic strain $\Gamma$ within the grain, an extended Voce hardening rule was applied as follows [5]:

$$
\tau^{s}=\tau_{0}^{s}+\left(+\theta_{1}^{s} \Gamma\right)\left(1-\exp \left(\frac{-\theta_{0}^{s} \Gamma}{\tau_{1}^{s}}\right)\right),
$$

where $\tau_{0}^{s}$, and $\tau_{1}^{s}$ are the initial and back-extrapolated CRSS, respectively. Further fitting parameters are $\theta_{0}^{s}$ and $\theta_{1}^{s}$, i.e. the initial and asymptotic hardening rates. Since the original EPSC model is suitable primarily for characterization of slip-dominated deformation [1], it was extended by Clausen et al. [5] in order to account for twin domain reorientation and associated stress relaxation.

\section{Results and discussion}

The neutron diffraction patterns obtained by axial detector ("bank 2") were evaluated by the convolutional multiple whole profile (CMWP) fitting method [16]. In this procedure the diffraction pattern is fitted by the sum of a background spline and the convolution of the instrumental pattern and the profiles related to crystallite (coherently scattering domain) size and dislocations. The crystallite size broadening can be neglected due to the large grain size of the specimens studied. The fitting and data evaluation procedure is described in detail in $[17,18]$, therefore only a short notice is given here. The line profile broadening caused by dislocations can be characterized by the mean-square-strain

$$
\left\langle\varepsilon_{g, L}^{2}\right\rangle \cong \frac{\rho \sum_{i=1}^{N} f_{i} \bar{C}_{h k l}^{(i)} b_{i}^{2}}{4 \pi} f(\eta),
$$

where $f(\eta)$ is the Wilkens function, $f_{i}$ are the fractions of the particular slip system families ${ }^{\ddagger}$. The average dislocation contrast factor $\bar{C}_{h k l}^{(i)}$ corresponding to the $i$-th slip system family can be expressed as

$$
\bar{C}_{h k l}^{(i)}=\bar{C}_{h k 0}^{(i)}\left(1+q_{1, i} z+q_{2, i} z^{2}\right),
$$

where $q_{1, i}$ and $q_{2, i}$ are two parameters depending on the anisotropic elastic constants and $z$ is a parameter depending on the lattice constant and the diffraction vector. $\bar{C}_{h k 0}^{(i)}$ is the average dislocation contrast factor of the $h k 0$ type reflections for the $i$-th slip system family. The outputs of the fitting procedure are the dislocation density $\rho$ and the parameters $q_{1}^{m}$ and $q_{2}^{m}$. The relation between the measured and the theoretical values for $q_{1}$ and $q_{2}$ (calculated in $\left.[17,19]\right)$ are given by the following equations:

$$
q_{1}^{m}=\sum_{i=1}^{11} f_{i} \bar{C}_{h k 0}^{(i)} b_{i}^{2} q_{1, i} / \sum_{i=1}^{11} f_{i} \bar{C}_{h k 0}^{(i)} b_{i}^{2},
$$

$\ddagger$ There are eleven slip system families considered. The notation, the Burgers vector, the slip plane and the number of slip systems for each hexagonal slip system family are listed in the paper by Dragomir and Ungár [17]

$$
q_{2}^{m}=\sum_{i=1}^{11} f_{i} \bar{C}_{h k 0}^{(i)} b_{i}^{2} q_{2, i} / \sum_{i=1}^{11} f_{i} \bar{C}_{h k 0}^{(i)} b_{i}^{2},
$$

and

$$
\sum_{i=1}^{11} f_{i}=1
$$

Since the equation system is underdetermined ( 3 equations, 11 unknown), the eleven values of $f_{j}$ cannot be determined from the three formulae in Eq. $(4 \mathrm{a}-\mathrm{c})$, therefore additional restrictions are made for $f_{j}$. The problem is simplified by grouping of the dislocations based on their Burgers vectors as $\langle a\rangle$ type, $\langle c\rangle$ type and $\langle c+a\rangle$ type. It is assumed that in each Burgers vector group the non-zero fractions are equal. This assumption reduces the number of variables to three, which are denoted by $f_{a}, f_{c}$ and $f_{c+a}$. A computer program was elaborated in order to determine the distribution of dislocations among the different slip system families from $q_{1}^{m}$ and $q_{2}^{m}$ [18]. The program referred to as Hexburger first selects some slip system families from $\langle a\rangle$ dislocation group and for these slip systems the weights are $f_{a}$. For other slip systems in this group the weights are zero. This procedure is also carried out for $\langle c\rangle$ and $\langle c+a\rangle$ Burgers vector groups where the non-zero weights are $f_{c}$ and $f_{c+a}$, respectively. Inserting the theoretical values of $\bar{C}_{h k 0}^{(i)}, b_{j}, q_{1, i}$ and $q_{2, i}$ into Eq. $(4 \mathrm{a}-\mathrm{c})$, the values of $f_{a}, f_{c}$ and $f_{c+a}$ are determined. If these fractions have positive values, the program stores them as one of the possible solutions. Finally, the positive solutions for the weights can be averaged for each slip system family, leading to the fractions of the eleven dislocation types. The fractions of the three Burgers vector groups are obtained by the summation of the fractions of the related slip system families.

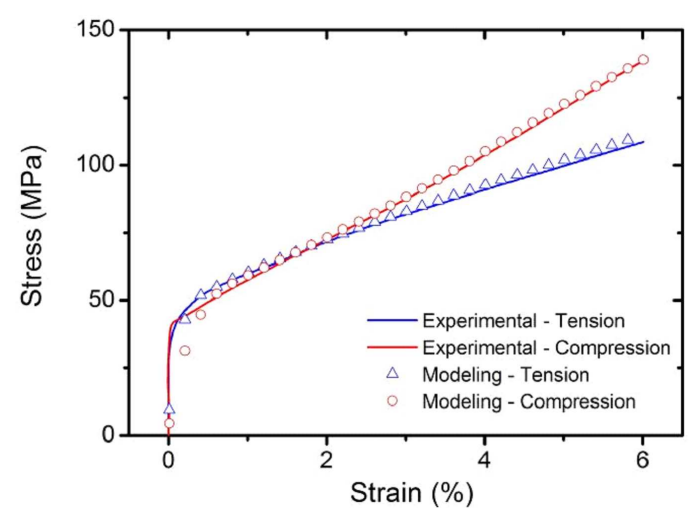

Fig. 1. The experimental and calculated (by EPSC) stress - strain curves for both tension and compression.

Both the deformation curves (Fig. 1) and the evolution of the dislocation density (Fig. 2) is different for the two loading modes. The dislocation density increases for both cases (Fig. 2), but above about $2 \%$ of applied strain the increment is higher for the compressed sample. This effect can be explained by the difference in twin growth between tension and compression. As it was shown in our recent work [20], in compression a rapid growth of nucle- 


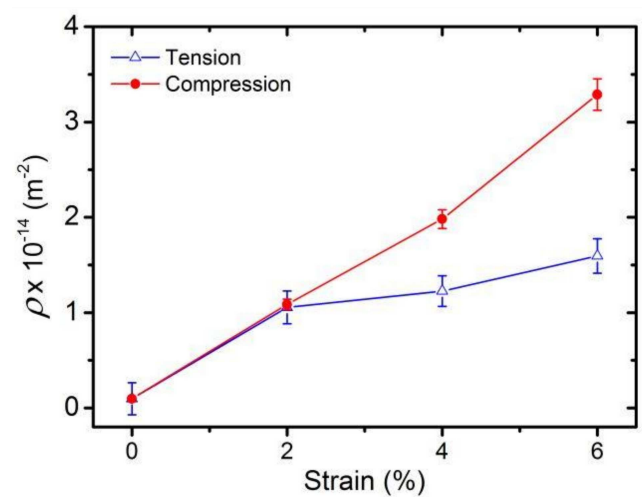

Fig. 2. Evolution of the dislocation density as a function of the applied strain for both tension and compression.

ated extension ( $\{10 \overline{1} 2\}$-type) twins takes place, contrary to the tension, where nucleation of several twin variants within a single grain is preferred at the expense of the twin growth (see also [21]). The twin growth is related to the concurrent activity of prismatic and basal $\langle a\rangle$ dislocations [22]. Furthermore, the dislocations crossing the parent-twin boundary can transmute to a less mobile type and this process yields an increase in the dislocation density owing to the reduced annihilation ability [6]. Finally, in grains, which are completely "consumed" by twins, activation of $\langle c+a\rangle$ slip is expected [23].

The evolution of the ratio of fractions of basal and prismatic $\langle a\rangle$ dislocations with increasing strain (Fig. 3) proves the key role of prismatic $\langle a\rangle$ slip in the macroscopic yield. The prismatic $\langle a\rangle$ slip seems a bit more active in tension, which is in good agreement with the observation of Agnew et al. [23]. It should be noted that from the point of view of the neutron diffraction profiles, the prismatic and pyramidal $\langle a\rangle$ dislocations contribute to the line broadening by similar manner. Making difference between them needs a deeper analysis, which is beyond the scope of this paper. Despite of considering only the prismatic $\langle a\rangle$ slip here, we do not exclude the possibility of the activity of pyramidal $\langle a\rangle$ slip.

A significant influence of the loading mode on the activity of particular dislocation types can be found in the case of second-order pyramidal dislocations (Fig. 3). Whereas in tension, the $\langle a\rangle /\langle c+a\rangle$ ratio is essentially unchanged, in compression a slightly higher fraction of $\langle c+a\rangle$ was observed above $2 \%$ strain. The main reason for such a behavior can be found again in the rapid twin growth in compression. When a grain completely changes his initial orientation as a consequence of the extension twinning, or the twin growth is limited (e.g. owing to the back-stresses induced by the neighboring grains [24]), the further deformation is substantiated by activation of a new mechanism. The dislocation slip in the second order $(\{11 \overline{2} 2\}\langle\overline{1} \overline{1} 23\rangle)$ pyramidal plane, accommodating strain along the crystal $c$-axis and offering five independent slip systems is the possibility, which was found out and studied both experimentally [7, 25] and theoretically [26].

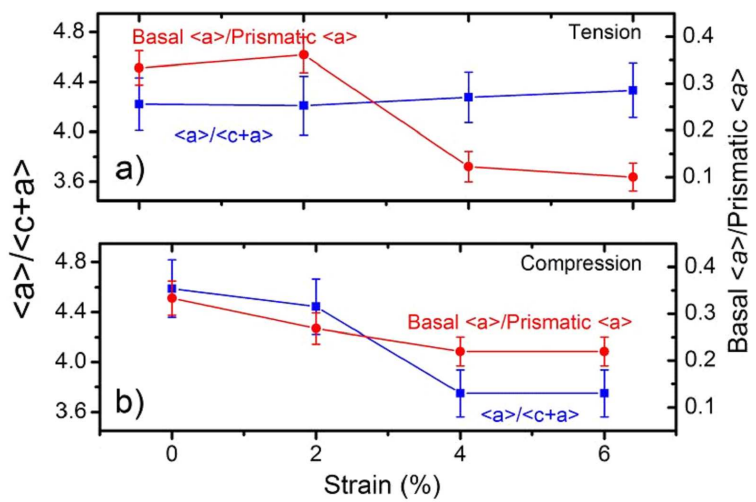

Fig. 3. Dependence of the ratios of the fraction of $\langle a\rangle$ type dislocations versus $\langle c+a\rangle$-type dislocations and fraction of basal $\langle a\rangle$ versus prismatic $\langle a\rangle$ dislocations on the applied strain for both loading modes, as evaluated by neutron diffraction peak profile analysis.

In the calculations of the EPSC model we incorporated twenty thousand grains with random texture distribution, basal, prismatic, first- and second order pyramidal slip and extension twinning were considered in the calculations. The initial fitting parameters are listed in the Table.

TABLE

Initial fitting parameters of EPSC model (see Eq. (1)).

\begin{tabular}{c|c|c|c|c}
\hline \hline \multirow{2}{*}{} & $\tau^{s}$ & $\tau_{1}^{s}$ & $\theta^{s}$ & $\theta_{1}^{s}$ \\
\cline { 2 - 5 } & \multicolumn{4}{|c}{$[\mathrm{MPa}]$} \\
\hline basal $\langle a\rangle$ & 4 & 2 & 200 & 50 \\
\hline prismatic $\langle a\rangle$ & 18 & 10 & 240 & 100 \\
\hline pyramidal $\langle a\rangle$ & 70 & 60 & 400 & 160 \\
\hline pyramidal $\langle c+a\rangle$ & 75 & 60 & 150 & 150 \\
\hline tensile twinning & 8 & 0 & 0 & 0
\end{tabular}

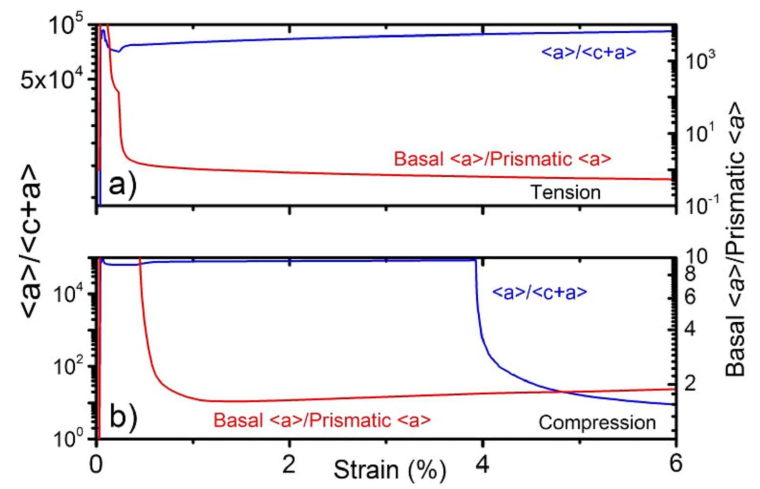

Fig. 4. Dependence of the ratios of the activity of $\langle a\rangle$ type dislocation slip versus $\langle c+a\rangle$-type dislocation slip and activity of basal $\langle a\rangle$ slip versus prismatic $\langle a\rangle$ slip on the applied strain for both loading modes, as calculated using EPSC model.

The theoretical stress-strain curves satisfactorily fit the experimental ones (Fig. 1). The model enables to calculate the activity of each deformation process considered 
as a function of the applied load. The activity ratios of basal $\langle a\rangle /$ prismatic $\langle a\rangle$ and $\langle a\rangle$ slip $/\langle c+a\rangle$ slip are depicted in Fig. 4 in order to compare them with the CMWP results. It is obvious that the trend of the theoretical and experimental curves is quite similar. Nevertheless, it should be kept in mind that the EPSC model describes the easiness (activity) of the glide, whereas the output of CMWP is the density of dislocations in the different slip systems. Furthermore, the initial dislocation configuration can be included into the model only indirectly through the CRSS and hardening parameters, whereas all CMWP results at non-zero strains are influenced by initial densities of the initial state. Thus, only qualitative conclusions from obtained results can be made.

\section{Conclusions}

The loading mode dependence of the evolution of the dislocation structure up to the strain of $6 \%$ was investigated using a concept based on the neutron diffraction line profile analysis and the EPSC modeling. The dislocation density was found to be higher in compression than in tension above $2 \%$ of applied strain. It was shown that the non-basal $\langle a\rangle$ slip plays a substantial role in the macroplasticity in both tension and compression. Slip in the second-order pyramidal system exhibited a dependence on the loading mode. The activity of $\langle c+a\rangle$ slip was significantly higher in compression than in tension. The EPSC model predicted well the experimentally observed behavior of particular slip modes.

\section{Acknowledgments}

The authors are grateful for the financial support of the Czech Science Foundation under the contract 14-36566G. J.Č. acknowledges the support from the Grant Agency of Charles University under contract Nr. 251715. J.G. acknowledges the support form the Hungarian Scientific Research Fund, OTKA, Grant No. K-109021.

This work has benefited from the use of the Lujan Neutron Scattering Center at LANSCE, funded by the US Department of Energy's Office of Basic Energy Sciences. Los Alamos National Laboratory is operated by Los Alamos National Security LLC under US DOE Contract DE-AC52-06NA25396.

\section{References}

[1] S.R. Agnew, O. Duygulu, Int. J. Plast. 21, 1161 (2005).

[2] M.R. Barnett, Z. Keshavarz, A.G. Beer, D. Atwell, Acta Mater. 52, 5093 (2004).
[3] A.H. Blake, C.H. Caceres, in: Magnesium Technol., Eds.: N. Neelamegghan, H.I. Kaplan, B.R. Powell, MMS, 2005, p. 403.

[4] C.H. Caceres, A. Blake, Phys. Status Solidi A 194, 147 (2002).

[5] B. Clausen, C.N. Tome, D.W. Brown, S.R. Agnew, Acta Mater. 56, 2456 (2008).

[6] A.L. Oppedal, H. El Kadiri, C.N. Tome, G.C. Kaschner, S.C. Vogel, J.C. Baird, M.F. Horstemeyer, Int. J. Plast. 30-31, 41 (2012).

[7] S. Sandloebes, Z. Pei, M. Friak, L.F. Zhu, F. Wang, S. Zaefferer, D. Raabe, J. Neugebauer, Acta Mater. 70, 92 (2014).

[8] P. Dobroň, J. Bohlen, F. Chmelík, P. Lukáč, D. Letzig, K.U. Kainer, Mater. Sci. Eng. A 462, 307 (2007).

[9] K. Hazeli, J. Cuadra, P.A. Vanniamparambil, A. Kontsos, Scr. Mater. 68, 83 (2013).

[10] O. Muránsky, M.R. Barnett, D.G. Carr, S.C. Vogel, E.C. Oliver, Acta Mater. 58, 1503 (2010).

[11] L. Balogh, D.W. Brown, P. Mosbrucker, F. Long, M.R. Daymond, Acta Mater. 60, 5567 (2012).

[12] T. Ungár, A.D. Stoica, G. Tichy, X.-L. Wang, Acta Mater. 66, 251 (2014).

[13] M.A.M. Bourke, D.C. Dunand, E. Ustundag, Appl. Phys. A 74, S1707 (2002).

[14] P.A. Turner, C.N. Tome, C.H. Woo, Philos. Mag. A 70, 689 (1994).

[15] C.J. Neil, J.A. Wollmershauser, B. Clausen, C.N. Tome, S.R. Agnew, Int. J. Plast. 26, 1772 (2010).

[16] G. Ribarik, T. Ungar, J. Gubicza, J. Appl. Crystallogr. 34, 669 (2001).

[17] I.C. Dragomir, T. Ungar, J. Appl. Crystallogr. 35, 556 (2002).

[18] K. Máthis, K. Nyilas, A. Axt, I. DragomirCernatescu, T. Ungár, P. Lukáč, Acta Mater. 52, 2889 (2004).

[19] P. Klimanek, R. Kužel, J. Appl. Crystallogr. 21, 59 (1988).

[20] J. Čapek, K. Máthis, B. Clausen, J. Stráská, P. Beran, P. Lukáš, Mater. Sci. Eng. A 602, 25 (2014).

[21] S.H. Park, J.H. Lee, B.G. Moon, B.S. You, J. Alloys Comp. 617, 277 (2014).

[22] B. Xu, L. Capolungo, D. Rodney, Scr. Mater. 68, 901 (2013).

[23] S.R. Agnew, D.W. Brown, C.N. Tome, Acta Mater. 54, 4841 (2006).

[24] M.R. Barnett, N. Stanford, A. Ghaderi, F. Siska, Acta Mater. 61, 7859 (2013).

[25] J.F. Stohr, J.P. Poirier, Philos. Mag. 25, 1313 (1972).

[26] T. Nogaret, W.A. Curtin, J.A. Yasi, L.G. Hector, Jr., D.R. Trinkle, Acta Mater. 58, 4332 (2010). 